\title{
Targeting interleukins in Alzheimer's disease
}

\section{By Kai-Jye Lou, Staff Writer}

Researchers at Charité-University Hospital Berlin and the University of Zurich have data showing that $\mathrm{p} 40$, a protein subunit shared by IL-12 and IL-23, could be a new therapeutic target in Alzheimer's disease. ${ }^{1}$ The group now is trying to better understand the downstream mediators of the pathway and wants to find an industry partner to test late-stage or marketed p40-targeting therapies, such as Johnson \& Johnson's psoriasis drug Stelara ustekinumab.

$\mathrm{p} 40$ is produced by microglia and is a protein subunit shared by IL-12 and IL-23. Microglia are the resident macrophages of the CNS, and aberrant phenotypes are linked to multiple neurodegenerative diseases, including Alzheimer's disease (AD). ${ }^{2}$ These CNS cells accumulate around $\beta$-amyloid $(\mathrm{A} \beta)$ plaques and are known to be a major source of proinflammatory cytokines, ${ }^{3}$ although their precise role in $\mathrm{AD}$ pathogenesis and progression is unclear. ${ }^{4}$

$\mathrm{A} \beta$ plaques are hallmarks of $\mathrm{AD}$ and trigger inflammatory responses. ${ }^{5}$

A research group co-led by Frank Heppner has been trying to characterize the role of microglia in $\mathrm{A} \beta$ pathology in $\mathrm{AD}$. Initially, it seemed there was at least no immediate role. In 2009, his group reported that ablation of the microglia cell population for four weeks had no observable effect on $A \beta$ plaque load and amyloid-associated neurotoxicity in two mouse models of AD. ${ }^{6}$

Now, however, his team has shown that targeting a cytokine subunit produced by aberrant microglia for at least 60 days reduces $A \beta$ plaque loads and reverses $\mathrm{AD}$ pathology.

In a transgenic mouse model of $\mathrm{AD}$, microglial production of $\mathrm{p} 40-$ as well as levels of Il-12 and Il-23-was greater than that in wild-type controls.

"We found that these molecules were upregulated in a distinct subpopulation of microglia in this mouse model of Alzheimer's and thus thought they may be associated with disease pathology," said Heppner, chair of the Department of Neuropathology at Charité-University Hospital Berlin. "Thus, we sought to also investigate what happens when we inhibit the signaling of these molecules in the mouse model-first genetically and then pharmacologically after seeing positive effects."
Peripheral delivery of an antibody against $\mathrm{p} 40$ decreased $\mathrm{A} \beta$ plaque burden in young mice compared with delivery of an isotype control. In the same model using aged mice, intracerebroventricular delivery of the p40-targeting antibody decreased both cognitive deficits and soluble $A \beta$ burden compared with what was seen in controls.

Behavioral assessments were not carried out in the young mice as the $\mathrm{AD}$-associated cognitive deficits in this model are not detectable until the animals are older. The group did not evaluate peripheral delivery of the $\mathrm{p} 40$-targeting antibody in the aged mice.

The link between $\mathrm{p} 40$ and $\mathrm{AD}$ also carried over to patients.

An analysis of cerebrospinal fluid (CSF) samples from 39 patients with $\mathrm{AD}$ and 20 non-AD controls showed an association between elevated levels of $\mathrm{p} 40$ and reduced cognitive performance $(p<0.05)$.

Results were published in Nature Medicine.

"Our study not only helps to confirm that inflammation is a crucial mediator of Alzheimer's pathophysiology but also shows what type of molecule to target in the immune system in this disease setting," said Heppner, a co-corresponding author. "Our results clearly show for the first time a strong association of IL-12 and IL-23 with Alzheimer's and identify p40 as a candidate therapeutic target for the disease."

"The data in their mouse model studies are very strong and show that targeted regulation of microglial function leads to improvements in Alzheimer's pathophysiology," added Terrence
- Terrence Town, Town, a professor of biomedical sciences and chair in regenerative medi-
cine at the Regenerative Medicine Institute at Cedars-Sinai Medical Center and the University of California, Los Angeles David Geffen School of Medicine. "They also looked at CSF samples from humans, lending support to the case that what they see in mice is also happening in humans."

"We look forward to seeing these preclinical results reproduced and confirmed with the hope that they will shed more light on the mechanistic role of inflammation in cognitive disorders and that they will help provide an informed foundation for successful translation to clinical therapeutic application for drugs like Stelara," said J\&J spokesperson Brian Kenney.

\section{p40 questions}

Stelara is marketed to treat plaque psoriasis and is under review to treat active psoriatic arthritis. The mAb also is in Phase III testing for Crohn's disease.

The other late-stage, p40-targeting mAb is Abbott Laboratories' Ozespa briakinumab, which is in Phase II testing for Crohn's disease. Abbott withdrew a BLA in the U.S. and an MAA in the EU for Ozespa to treat psoriasis in January 2011 after the agencies asked for additional data and analyses.

"This isn't just another study where you look at the results and in 15 years, maybe you will have something in the clinic," said Richard Ransohoff, director of the Neuroinflammation Research Center at the 
Cleveland Clinic. "Instead, this is a study where reagents against the newly identified target are already available. With careful thought and trial design, they could begin testing their therapeutic hypothesis in a translational program."

An open question is how to best deliver a mAb-based therapy across the blood brain barrier (BBB).

"When we applied our antibody peripherally in the mouse model, we found that it had a significant effect in reducing plaque burden in the brain, which suggests that sufficient amounts of the antibody could be getting across the blood brain barrier and that peripheral application might be enough," Heppner told SciBX.

Town said that intrathecal delivery is another delivery option and added that other research groups already are working on strategies to deliver $\mathrm{mAbs}$ and other large-molecule therapeutics across the BBB and into the CNS.

One such approach is the molecular Trojan horse drug delivery technology being developed by ArmaGen Technologies Inc. ${ }^{78}$ The technology involves creating a fusion protein of a therapeutic compound and a Trojan horse molecule that targets surface receptors on endothelial cells to facilitate transport across the BBB.

Another strategy, being developed by researchers at Cornell University, involves temporarily agonizing adenosine receptors in the brain's vasculature to loosen the BBB., ${ }^{9,10}$

Alternatively, small molecule inhibitors of IL-12 and IL-23 could be easier to get across the BBB than antibodies.

At least one company-Synta Pharmaceuticals Corp.-has small molecule inhibitors of IL-12 and IL-23 in preclinical development to treat various autoimmune and inflammatory diseases. The company also previously developed apilimod, a small molecule that inhibits IL-12 and IL-23 production, to treat plaque psoriasis, Crohn's disease, common variable immunodeficiency (CVID) and rheumatoid arthritis (RA). The compound failed in Phase II trials in all four indications.

Michael Heneka, a professor of clinical neurosciences at the University of Bonn, thinks it is still too early to say whether targeting p40 will pan out in the $\mathrm{AD}$ setting and said the real question is when-and not how-to inhibit p40 in AD.

"Further studies will have to identify the precise time course of this particular immunological pathway in AD," he told SciBX. "I would want to see studies in human samples to determine at what stages of $A D$ and pre-AD this cytokine signaling pathway is active and how signaling in the pathway changes at different stages of the disease. I would also like to see an assessment of how this pathway affects different brain regions and a study to determine this pathway's relevance to sporadic and familial cases of AD."

Ransohoff said it will be important to determine the cells that respond to IL-12 and IL-23 signaling in the AD setting and to further elucidate how signaling through these cytokines contributes to disease pathology.

Town said additional validation studies in postmortem brain tissue from patients with $\mathrm{AD}$ could help determine the cells responsible for producing IL-12 and IL-23 in close vicinity to the plaque lesions.

Heppner's group now is trying to replicate the study results using a different mouse AD model with later disease onset. His group also is continuing with basic science studies to better characterize the function of p40, IL-23 and IL-23 downstream of microglia.

"What we want to do is to study downstream effects and mechanisms in this pathway," he told SciBX. "The other axis we are interested in is finding a partner from industry to help set up a clinical trial, for example, to evaluate an existing p40-targeting antibody or similar compounds in Alzheimer's patients."

The University of Zurich and Charité-University Hospital Berlin have cofiled a patent covering modulators of IL-12 and/or IL-23 to treat and prevent $\mathrm{AD}$. The work is available for licensing from Unitectra, the technology transfer office of the University of Zurich.

Lou, K.-J. SciBX 5(49); doi:10.1038/scibx.2012.1276

Published online Dec. 20, 2012

\section{REFERENCES}

1. Von Berg, J. et al. Nat. Med.; published online Nov. 25, 2012; doi:10.1038/nm.2965

Contact: Frank L. Heppner, Charité-University Hospital Berlin, Berlin, Germany

e-mail: frank.heppner@charite.de

Contact: Burkhard Becher, University of Zurich, Zurich, Switzerland e-mail: becher@immunology.uzh.ch

2. Perry, V.H. et al. Nat. Rev. Neurol. 6, 193-201 (2010)

3. Streit, W.J. et al. J. Neuroinflammation 1, 14 (2004)

4. Jucker, M. \& Heppner, F.L. Neuron 59, 8-10 (2008)

5. Hickman, S.E. et al. J. Neurosci. 28, 8354-8360 (2008)

6. Grathwohl, S.A. et al. Nat. Neurosci. 12, 1361-1363 (2009)

7. Boado, R.J. Drug News Perspect. 21, 489-503 (2008)

8. Fulmer, T. SciBX 1(41); doi:10.1038/scibx.2008.991

9. Carman, A.J. et al. J. Neurosci. 31, 13272-13280 (2011)

10. Osherovich, L. SciBX 4(39); doi:10.1038/scibx.2011.1080

\section{COMPANIES AND INSTITUTIONS MENTIONED}

Abbott Laboratories (NYSE:ABT), Abbott Park, III. ArmaGen Technologies Inc., Santa Monica, Calif. Cedars-Sinai Medical Center, Los Angeles, Calif. Charité-University Hospital Berlin, Berlin, Germany Cleveland Clinic, Cleveland, Ohio Cornell University, Ithaca, N.Y.

Johnson \& Johnson (NYSE:JNJ), New Brunswick, N.J.

Synta Pharmaceuticals Corp. (NASDAQ:SNTA), Lexington, Mass. Unitectra, Zurich, Switzerland

University of Bonn, Bonn, Germany

University of California, Los Angeles David Geffen School of

Medicine, Los Angeles, Calif.

University of Zurich, Zurich, Switzerland 